\title{
On the decoding of Barnes-Wall lattices
}

\author{
Vincent Corlay ${ }^{\dagger, *}$, Joseph J. Boutros ${ }^{\ddagger}$, Philippe Ciblat ${ }^{\dagger}$, and Loïc Brunel* \\ $\dagger$ Telecom Paris,Institut Polytechnique de Paris, 91120 Palaiseau, France, v.corlay@ fr.merce.mee.com \\ $\ddagger$ Texas A\&M University, Doha, Qatar, *Mitsubishi Electric R\&D Centre Europe, Rennes, France
}

\begin{abstract}
We present new efficient recursive decoders for the Barnes-Wall lattices based on their squaring construction. The analysis of the new decoders reveals a quasi-quadratic complexity in the lattice dimension and a quasi-linear complexity in the listsize. The error rate is shown to be close to the universal lower bound in dimensions 64 and 128.
\end{abstract}

\section{INTRODUCTION}

Barnes-Wall $(B W)$ lattices were one of the first series discovered with an infinitely increasing fundamental coding gain [2]. This series includes dense lattices in lower dimensions such as $D_{4}, E_{8}, \Lambda_{16}$ [5], and is deeply related to Reed-Muller codes [9][17]: $B W$ lattices admit a Construction D based on these codes. Multilevel constructions attracted the recent attention of researchers, mainly Construction $\mathrm{C}^{*}$ [3], where lattice and non-lattice constellations are made out of binary codes. One of the important challenges is to develop lattices with a reasonable-complexity decoding where a fraction of the fundamental coding gain is sacrificed in order to achieve a lower kissing number. $B W$ lattices are attractive in this sense. For instance the lattice $B W_{128}$, with an equal fundamental coding gain as $N e b e_{72}$ [20], sacrifices $1.5 \mathrm{~dB}$ of its fundamental coding gain with respect to $M W_{128}$ [8] while the kissing number is reduced by a factor of 200 .

Several algorithms have been proposed to decode $B W$ lattices. Forney introduced an efficient maximum-likelihood decoding (MLD) algorithm in [9] for the low dimension instances of these lattices based on their trellis representation. Nevertheless, the complexity of this algorithm is exponential in the dimension and intractable for $n>32$ : e.g. decoding in $B W_{64}$ involves $2 \cdot 2^{24}+2 \cdot 2^{16}$ decoders of $B W_{16}$ and decoding in $B W_{128}$ involves $2 \cdot 2^{48}+2 \cdot 2^{32}$ decoders of $B W_{32}$ (using the two-level squaring construction to build the trellis, see [9, Section IV.B]). Later, [19] proposed the first boundeddistance decoders (BDD) running in polynomial time: a parallelisable decoder of complexity $O\left(n^{2}\right)$ and another sequential decoder of complexity $O\left(n \log ^{2}(n)\right)$. The parallel decoder was generalized in [14] to work beyond the packing radius, still in polynomial time. It is discussed later in the paper. The sequential decoder uses the $B W$ multilevel construction to perform multistage decoding: each of the $\approx \log (n)$ levels is decoded with a Reed-Muller decoder of complexity $n \log (n)$. This decoder was also further studied, in [15], to design practical schemes for communication over the AWGN channel. The performance of this sequential decoder is far from MLD. A simple information-theoretic argument explains why multistage decoding of $B W$ lattices cannot be efficient: the rates of some component Reed-Muller codes exceed the channel capacities of the corresponding levels [13][28]. As a result, no $B W$ decoders, being both practical and quasi-optimal on the Gaussian channel, have been designed and executed for dimensions greater than 32 .

We present new decoders for $B W$ lattices based on their $(u, u+v)$ construction [17]. We particularly consider this construction as a squaring construction [9] to establish a new recursive BDD (Algorithm 2, Section III-A), new recursive list decoders (Algorithms 3 and 5, Sections IV-B and IV-C), and their complexity analysis as stated by Theorems 2-4. As an example, Algorithm 5 decodes $B W_{64}$ and $B W_{128}$ with a performance close to the universal lower bound on the coding gain of any lattice and with a reasonable complexity almost quadratic in the lattice dimension.

\section{PRELIMINARIES}

Lattice. A lattice $\Lambda$ is a discrete additive subgroup of $\mathbb{R}^{n}$. For a rank- $n$ lattice in $\mathbb{R}^{n}$, the rows of a $n \times n$ generator matrix $G$ constitute a basis of $\Lambda$ and any lattice point $x$ is obtained via $x=z G$, where $z \in \mathbb{Z}^{n}$. The squared minimum Euclidean distance of $\Lambda$ is $d(\Lambda)=(2 \rho(\Lambda))^{2}$, where $\rho(\Lambda)$ is the packing radius. The number of lattice points located at a distance $\sqrt{d(\Lambda)}$ from the origin is the kissing number $\tau(\Lambda)$. The fundamental volume of $\Lambda$, i.e. the volume of its Voronoi cell and its fundamental parallelotope, is denoted by $\operatorname{vol}(\Lambda)$. The fundamental coding gain $\gamma(\Lambda)$ is given by the ratio $\gamma(\Lambda)=d(\Lambda) / \operatorname{vol}(\Lambda)^{\frac{2}{n}}$. The squared Euclidean distance between a point $y \in \mathbb{R}^{n}$ and a lattice point $x \in \Lambda$ is denoted $d(x, y)$. Accordingly, the squared distance between $y \in \mathbb{R}^{n}$ and the closest lattice point of $\Lambda$ is $d(y, \Lambda)$.

For lattices, the transmission rate used with finite constellations is meaningless. Poltyrev introduced the generalized capacity [22], the analog of Shannon capacity for lattices. The Poltyrev limit corresponds to a noise variance of $\sigma_{\max }^{2}=\operatorname{det}(\Lambda)^{\frac{2}{n}} /(2 \pi e)$ and the point error rate is evaluated with respect to the distance to Poltyrev limit, i.e. $\sigma_{\max }^{2} / \sigma^{2}$.

BDD, list-decoding, and MLD. Given a lattice $\Lambda$, a radius $r>0$, and any point $y \in \mathbb{R}^{n}$, the task of a decoder is to determine all points $x \in \Lambda$ satisfying $d(x, y) \leq r^{2}(\Lambda)$. If $r<\rho(\Lambda)$, there is either no point or a unique point found and the decoder is known as BDD. Additionally, if $d(x, y)<\rho^{2}(\Lambda)$, we say that $y$ is within the guaranteed error-correction radius of the lattice. If $r \geq \rho(\Lambda)$, there may be more than one point in the sphere. In this case, the process is called list-decoding rather than BDD. When list-decoding is used, lattice points within the sphere are enumerated and the decoded lattice point is the closest to $y$ among them. 
MLD simply refers to finding the closest lattice point in $\Lambda$ to any point $y \in \mathbb{R}^{n}$. If list-decoding is used, MLD is equivalent to choosing a decoding radius equal to $R(\Lambda)$.

Coset decomposition of a lattice. Let $\Lambda$ and $\Lambda^{\prime}$ be two lattices such that $\Lambda^{\prime} \subseteq \Lambda$. If the order of the quotient group $\Lambda / \Lambda^{\prime}$ is $q$, then $\Lambda$ can be expressed as the union of $q$ cosets of $\Lambda^{\prime}$. We denote by $\left[\Lambda / \Lambda^{\prime}\right]$ a system of coset representatives for this partition. It follows that $\Lambda=\bigcup_{x_{i} \in\left[\Lambda / \Lambda^{\prime}\right]} \Lambda^{\prime}+x_{i}=\Lambda^{\prime}+\left[\Lambda / \Lambda^{\prime}\right]$. The $B W$ lattices. Let the scaling-rotation operator $R(2 n)$ in dimension $2 n$ be defined by the application of the $2 \times 2$ matrix

$$
R(2)=\left[\begin{array}{rr}
1 & 1 \\
1 & -1
\end{array}\right]
$$

on each pair of components. I.e. the scaling-rotation operator is $R(2 n)=I_{n} \otimes R(2)$, where $I_{n}$ is the $n \times n$ identity matrix and $\otimes$ the Kronecker product. For $\Lambda \subset \mathbb{R}^{2 n}$ with generator matrix $G$, the lattice generated by $G \cdot R(2 n)$ is denoted $R \Lambda$.

Definition 1 (The squaring construction of $B W_{2 n}$ [9]). The $B W$ lattices in dimension $2 n$ are obtained by the following recursion:

$B W_{2 n}=\left\{(\underbrace{v_{1}^{\prime}+m}_{u_{1} \in B W_{n}}, \underbrace{v_{2}^{\prime}+m}_{u_{2} \in B W_{n}}), v_{i}^{\prime} \in R B W_{n}, m \in\left[B W_{n} / R B W_{n}\right]\right\}$, with initial condition $B W_{2}=\mathbb{Z}^{2}$.

Using this construction, it is easily seen that $d\left(B W_{2 n}\right)=$ $d\left(R B W_{n}\right)=2 d\left(B W_{n}\right)$ and the fundamental coding gain increases infinitely as $\gamma\left(B W_{n}\right)=\sqrt{2} \cdot \gamma\left(B W_{n / 2}\right)=\sqrt{n / 2}$ [9]. Note that the squaring construction can be expressed under the form of the Plotkin $(u, u+v)$ construction [21]:

$$
\begin{aligned}
B W_{2 n} & =\left\{\left(v_{1}^{\prime}+m, v_{2}^{\prime}+m\right), v_{i}^{\prime} \in R B W_{n}, m \in\left[B W_{n} / R B W_{n}\right]\right\}, \\
& =\left\{(v_{1}^{\prime}+m, \underbrace{v_{1}^{\prime}+v_{2}}_{v_{1}^{\prime}}+m)\right\}=\left\{\left(u_{1}, u_{1}+v_{2}\right)\right\} .
\end{aligned}
$$

\section{A. The new $B D D$}

\section{BOUNDED-DISTANCE $B W$ DECODING}

Given a point $y=\left(y_{1}, y_{2}\right) \in \mathbb{R}^{2 n}$ to be decoded, a wellknown algorithm [26]|7] for a code obtained via the $(u, u+v)$ construction is to first decode $y_{1}$ as $u_{1}$, and then decode $y_{2}-u_{1}$ as $v_{2} 1$. Our lattice decoder, Algorithm 1 is double-sided since we also decode $y_{2}$ as $u_{2}$ and then $y_{1}-u_{2}$ as $v_{2}$ : the decoder is based on the squaring construction. The main idea exploited by the algorithm is that if there is too much noise on one side, e.g. $y_{1}$, then there is less noise on the other side, e.g. $y_{2}$, and vice versa.

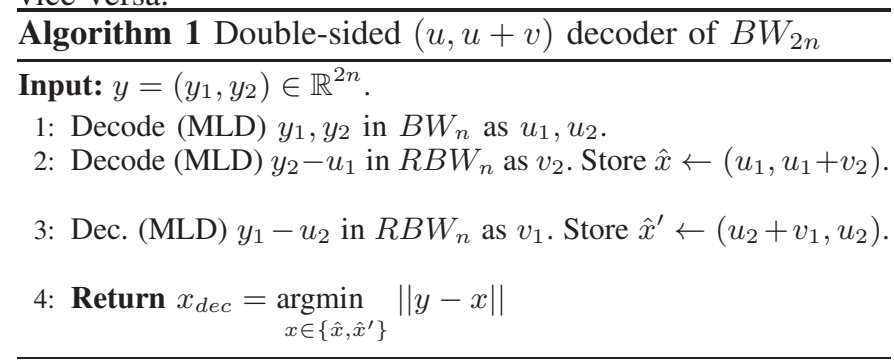

Theorem 1. Let $y$ be a point in $\mathbb{R}^{2 n}$ such that $d\left(y, B W_{2 n}\right)$ is less than $\rho^{2}\left(B W_{2 n}\right)$. Then, Algorithm $\square$ outputs the closest lattice point $x \in B W_{2 n}$ to $y$.

\footnotetext{
${ }^{1}$ The standard decoder for $(u, u+v)$ has a second round: once $v_{2}$ is decoded $u_{1}$ is re-decoded based on the two estimates $y_{1}$ and $y_{2}-v_{2}$.
}

Proof. If $\left(x_{1}, x_{2}\right) \in B W_{2 n}$, then $x_{1}, x_{2} \in B W_{n}$. Also, we have $\left\|\left(y_{1}, y_{2}\right)\right\|^{2}=\left\|y_{1}\right\|^{2}+\left\|y_{2}\right\|^{2}$. So if $d\left(y, B W_{2 n}\right)<\rho^{2}\left(B W_{2 n}\right)$, then at least one among the two $y_{i}$ is at a distance smaller than $\frac{\rho^{2}\left(B W_{2 n}\right)}{2}=\rho^{2}\left(B W_{n}\right)$ from $B W_{n}$. Therefore, at least one of the two $u_{i}$ is correct.

Assume (without loss of generality) that $u_{1}$ is correct. We have $d\left(y_{2}-u_{1}, R B W_{n}\right)<\rho^{2}\left(B W_{2 n}\right)=\rho^{2}\left(R B W_{n}\right)$. Therefore, $y_{2}-u_{1}$ is also correctly decoded.

As a result, among the two lattice points stored, at least one is the closest lattice point to $y$.

Note that the $B W_{n}$ decoder in the previous proof got exploited up to $\rho^{2}\left(B W_{n}\right)$ only. Consequently, Algorithm 1 should exceed the performance predicted by Theorem 1 given that step 1 is MLD.

Algorithm 1 can be generalized into the recursive Algorithm 2 where Steps 4, 5, and 6 of the latter algorithm replace Steps 1, 2, and 3 of Algorithm 1 respectively. This algorithm is similar to the parallel decoder of [19]. The main difference is that [19] uses the automorphism group of $B W_{2 n}$ to get four candidates at each recursion whereas we use the squaring construction to generate only two candidates. Nevertheless, both our algorithm and [19] use four recursive calls at each recursive section and have the same asymptotic complexity.

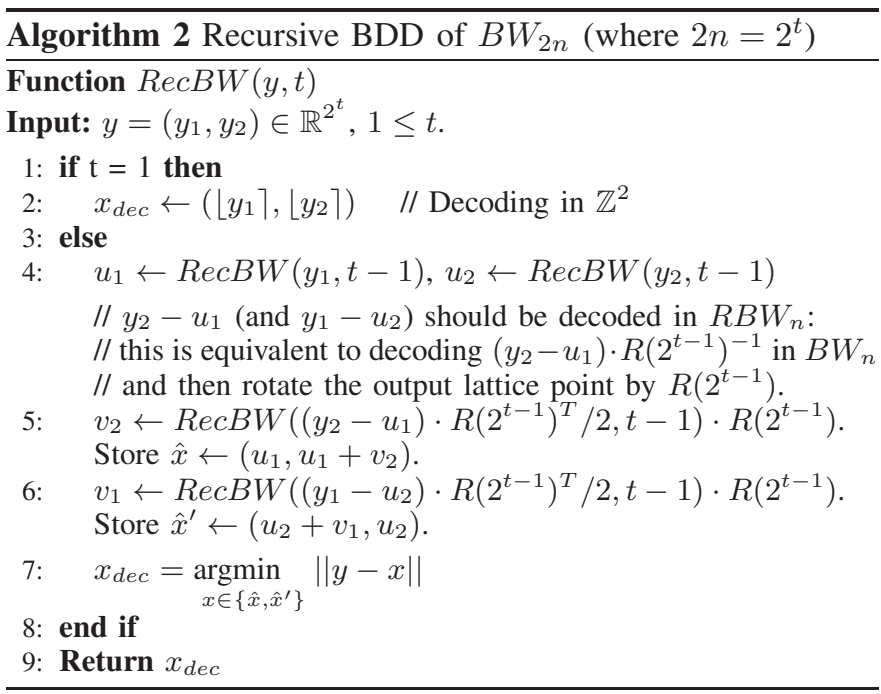

Theorem 2. Let $n$ be the dimension the lattice $B W_{n}$ to be decoded. The complexity of Algorithm 2 is $O\left(n^{2}\right)$.

Proof. Let $\mathfrak{C}(n)$ be the complexity of the algorithm for $n=2^{t}$. We have $\mathfrak{C}(n)=4 \mathfrak{C}(n / 2)+O(n)=O\left(n^{2}\right)$.

\section{B. Performance on the Gaussian channel}

In the appendix (see Section VII-A), we show via an analysis of the effective error coefficient of Algorithm 2 that the loss in performance of this algorithm compared to MLD (in $\mathrm{dB}$ ) is expected to grow linearly with $n$.

Our simulations show that there is a loss of $\approx 0.25 \mathrm{~dB}$ for $n=16, \approx 0.5 \mathrm{~dB}$ for $n=32, \approx 1.25 \mathrm{~dB}$ for $n=64$ (compare $\aleph=1$ and $\aleph=20$ on Figure 1 and $\approx 2.25 \mathrm{~dB}$ for $n=128$. As a result, this BDD is not suited for effective decoding of 
$B W$ lattices on the Gaussian channel. However, it is essential for building efficient decoders as shown in the next section.

\section{LIST-DECODING OF $B W$ LATTICES BEYOND THE PACKING RADIUS}

Let $L\left(\Lambda, r^{2}\right)$ be the maximum number of lattice points of $\Lambda$ within a sphere of radius $r$ around any $y \in \mathbb{R}^{n}$. If $\Lambda=B W_{n}$ we write $L\left(n, r^{2}\right)$. The following lemma is proved in [14].

Lemma 1. The list size of the $B W_{n}$ lattices is bounded as [14]:

- $L\left(n, r^{2}\right) \leq \frac{1}{2 \epsilon}$ if $r^{2} \leq d\left(B W_{n}\right)(1 / 2-\epsilon), 0<\epsilon \leq 1 / 4$.

- $L\left(n, r^{2}\right)=2 n$ if $r^{2}=d\left(B W_{n}\right) / 2$.

- $L\left(n, r^{2}\right) \leq 2 n^{16 \log _{2}(1 / \epsilon)}$ if $r^{2} \leq d\left(B W_{n}\right)(1-\epsilon)$, $0<\epsilon \leq 1 / 2$.

[14] also shows that the parallel BDD of [19], which uses the automorphism group of $B W_{n}$, can be slightly modified to output a list of all lattice points lying at a squared distance $r^{2}=d\left(B W_{n}\right)(1-\epsilon), \forall \epsilon>0$, from any $y \in \mathbb{R}^{n}$ in time $O\left(n^{2}\right) \cdot L\left(n, r^{2}\right)^{2}$. With Lemma 1 this becomes $n^{O(\log (1 / \epsilon))}$. This result is of theoretical interest: it shows that there exists a polynomial time algorithm in the dimension for any radius bounded away from the minimum distance. However, the quadratic dependence in the list-size is a drawback: finding an algorithm with quasi-linear dependence in the list-size is stated as an open problem in [14].

In the following, we show that if we use the squaring construction rather than the automorphism group of $B W_{n}$ for list-decoding, it is possible to get a quasi-linear complexity in the list size. This enables to get a practical list-decoding algorithm up to $n=128$.

1) Some notations: Notice that $L\left(n, r^{2}\right)=$ $L\left(R B W_{n}, 2 r^{2}\right)$, e.g. both are equal to $2 n$ if $r^{2}=d\left(B W_{n}\right) / 2$. It is therefore convenient to consider the relative squared distance as in [14]: $\delta(x, y)=\frac{d(x, y)}{d(\Lambda)}, x \in \Lambda$ 2. Then, if we define $l\left(\Lambda, r^{2} / d(\Lambda)\right)=L\left(\Lambda, r^{2}\right)$ this yields for instance $l(n, 1 / 2)=l\left(B W_{n}, 1 / 2\right)=l\left(R B W_{n}, 1 / 2\right)=2 n$. The relative squared radius is defined as the quantity $r^{2} / d(\Lambda)$. For the rest of this section, $\delta$ is the relative squared radius considered for decoding. Let $y=\left(y_{1}, y_{2}\right) \in \mathbb{R}^{2 n}$ and $x=\left(u_{1}, u_{1}+v_{2}\right)=\left(u_{2}+v_{1}, u_{2}\right) \in B W_{2 n}$ be any lattice point where $\delta(x, y) \leq \delta$. We recall that for BDD of $B W_{n}$ we have $\delta=1 / 4$.

The following lemma is trivial, but convenient to manipulate distances.

Lemma 2. (Lemma 2.1 in [14])

Let $y=\left(y_{1}, y_{2}\right) \in \mathbb{R}^{2 n}$ and $x=\left(u_{1}, u_{1}+v_{2}\right) \in B W_{2 n}$. Then,

$$
\delta(x, y)=\delta\left(u_{1}, y_{1}\right) / 2+\delta\left(v_{2}, y_{2}-u_{1}\right) .
$$

2) List-decoding with $r^{2}<9 / 16 d\left(B W_{n}\right)$ : Assume that the squared norm of the noise is $r^{2}$ and $\delta=\left(r^{2}+\epsilon\right) / d\left(B W_{n}\right)$. Consider $d(x, y)=d\left(u_{1}, y_{1}\right)+d\left(u_{2}, y_{2}\right)$. We split the possible

\footnotetext{
${ }^{2}$ Here, $\Lambda$ should be the "smallest" lattice to which $x$ belongs: e.g. take $u \in B W_{n}$. We also have $u \in R B W_{n}$ but $\Lambda$ should be $B W_{n}$.
}

situations into two main cases (similarly to Steps 2-3 of Algorithm [1]: $d\left(u_{1}, y_{1}\right) \leq r^{2} / 2$ and $d\left(u_{1}, y_{1}\right)>r^{2} / 2$. For the first case, $y_{1}$ should be list-decoded in $B W_{n / 2}$, and for each $u_{1} \in B W_{n / 2}$ in the resulting list, $y_{2}-u_{1}$ should be list-decoded in $R B W_{n / 2}$. Regarding the noise repartition, one can get the following two extreme configurations (but not simultaneously): $d\left(u_{1}, y_{1}\right)=r^{2} / 2$, i.e. $\delta\left(u_{1}, y_{1}\right)=\delta$ and $d\left(v_{2}, y_{2}-u_{1}\right)=r^{2}$, i.e. $\delta\left(v_{2}, y_{2}-u_{1}\right)=\delta$. Consequently, without any advanced strategy, the relative squared decoding radius to list-decode in $B W_{n / 2}$ and $R B W_{n / 2}$ should be $\delta$. The maximum of the product of the two resulting list-sizes, which is a key element in the complexity analysis below, is $l(n / 2, \delta)^{2}$. In order to reduce this number, we split this first case (i.e. $\left.d\left(u_{1}, y_{1}\right) \leq r^{2} / 2\right)$ into two sub-cases. Let $0 \leq a^{\prime} \leq r^{2} / 2$.

- $0 \leq d\left(u_{1}, y_{1}\right)<a^{\prime}$ and $r^{2} / 2<d\left(v_{2}, y_{2}-u_{1}\right) \leq r^{2}$ : then, $y_{1}$ should be list-decoded in $B W_{n / 2}$ with a relative squared radius $a_{1}=a^{\prime} / d\left(B W_{n / 2}\right)$ and $y_{2}-u_{1}$ list decoded in $R B W_{n / 2}$ with a relative squared radius $\delta$.

- $a^{\prime} \leq d\left(u_{1}, y_{1}\right) \leq r^{2} / 2$ and $r^{2} / 2<d\left(v_{2}, y_{2}-u_{1}\right) \leq$ $r^{2}-a^{\prime}$ : then, $y_{1}$ should be list-decoded in $B W_{n / 2}$ with a relative squared radius $\delta$ and $y_{2}-u_{1}$ list-decoded in $R B W_{n / 2}$ with a relative squared radius $a_{2}=\left(r^{2}-\right.$ $\left.a^{\prime}\right) / d\left(R B W_{n / 2}\right)$.

The size of the two resulting lists are bounded by $l\left(n / 2, a_{1}\right)$. $l\left(R B W_{n / 2}, \delta\right)$ and $l(n / 2, \delta) \cdot l\left(R B W_{n / 2}, a_{2}\right)$. Consequently, if we choose $a_{1}=a_{2}=a$, i.e. $a=2 / 3 \delta$, the two bounds are equal. The maximum number of candidates to consider becomes $2 l(n / 2, \delta) \cdot l(n / 2, a)$ which is likely to be much smaller than $l(n / 2, \delta)^{2}$, the bound obtained without the splitting strategy. The second case (i.e. $\left.d\left(u_{1}, y_{1}\right)>r^{2} / 2\right)$ is identical by symmetry.

This analysis yields Algorithm 3 listed below. The "removing step" (10 in bold) is added to ensure that a list with no more than $l(n, \delta)$ elements is returned by each recursive call. The maximum number of points to process by this removing step is $4 l(n / 2, \delta) l(n / 2, a)$. Regarding Step 11, using the classical Merge Sort algorithm, it can be done in $O(n \cdot l(n, \delta) \log (l(n, \delta)))$ operations (see App. VII-B).

The following theorem shows that we get an algorithm of quasi-linear complexity in the list size $l(n, \delta)$.

Theorem 3. Given any point $y \in \mathbb{R}^{n}$ and $1 / 4 \leq \delta<9 / 16$, Algorithm 3 outputs the list of all lattice points in $B W_{n}$ lying within a sphere of relative squared radius $\delta$ around $y$ in time:

- $O\left(n^{2} \log n\right) l(n, \delta)+O(n \log n) l(n, \delta) \log l(n, \delta)$ if $1 / 4 \leq \delta<3 / 8$

- $O\left(n^{1+\log _{2}(1+l(n, 2 / 3 \delta))} \log n\right) l(n, \delta) l(n, 2 / 3 \delta)+$ $O\left(n^{\log _{2}(1+l(n, 2 / 3 \delta))}\right) l(n, \delta) \log l(n, \delta)$ if $3 / 8 \leq \delta<9 / 16$.

Proof. Let $\mathfrak{C}(n, \delta)$ be the complexity of Algorithm 3 We have

$$
\begin{gathered}
\mathfrak{C}(n, \delta) \leq \underbrace{(2+2 l(n / 2, a)) \mathfrak{C}(n / 2, \delta)}_{\text {Numb. recursive calls with } \delta}+\underbrace{(2+2 l(n / 2, \delta)) \mathfrak{C}(n / 2, a)}_{\text {Numb. recursive calls with } a}+ \\
\underbrace{4 l(n / 2, \delta) l(n / 2, a) O(n)}_{\text {removing }}+\underbrace{O(n \cdot l(n, \delta) \log (l(n, \delta)))}_{\text {Merge Sort }} .
\end{gathered}
$$


If $\delta<3 / 8$, then $l(n, a) \leq 1$ and $\mathfrak{C}(n / 2, a) \leq$ $\mathfrak{C}(n / 2,1 / 4)=O\left((n / 2)^{2}\right)$ (the complexity of Algorithm 2]). (2) becomes

$$
\mathfrak{C}(n, \delta)=O\left(n^{2} \log n\right) l(n, \delta)+O(n \log n) l(n, \delta) \log l(n, \delta) .
$$

If $\delta<9 / 16$, then $\mathfrak{C}(n / 2, a)<\mathfrak{C}(n / 2,3 / 8)$. Using (3) for $\mathfrak{C}(n / 2,3 / 8)$, 2) becomes

$$
\begin{aligned}
& \mathfrak{C}(n, \delta)=O\left(n^{1+\log _{2}(1+l(n, 2 / 3 \delta))} \log n\right) l(n, \delta) l(n, 2 / 3 \delta)+ \\
& O\left(n^{\log _{2}(1+l(n, 2 / 3 \delta))}\right) l(n, \delta) \log l(n, \delta)
\end{aligned}
$$
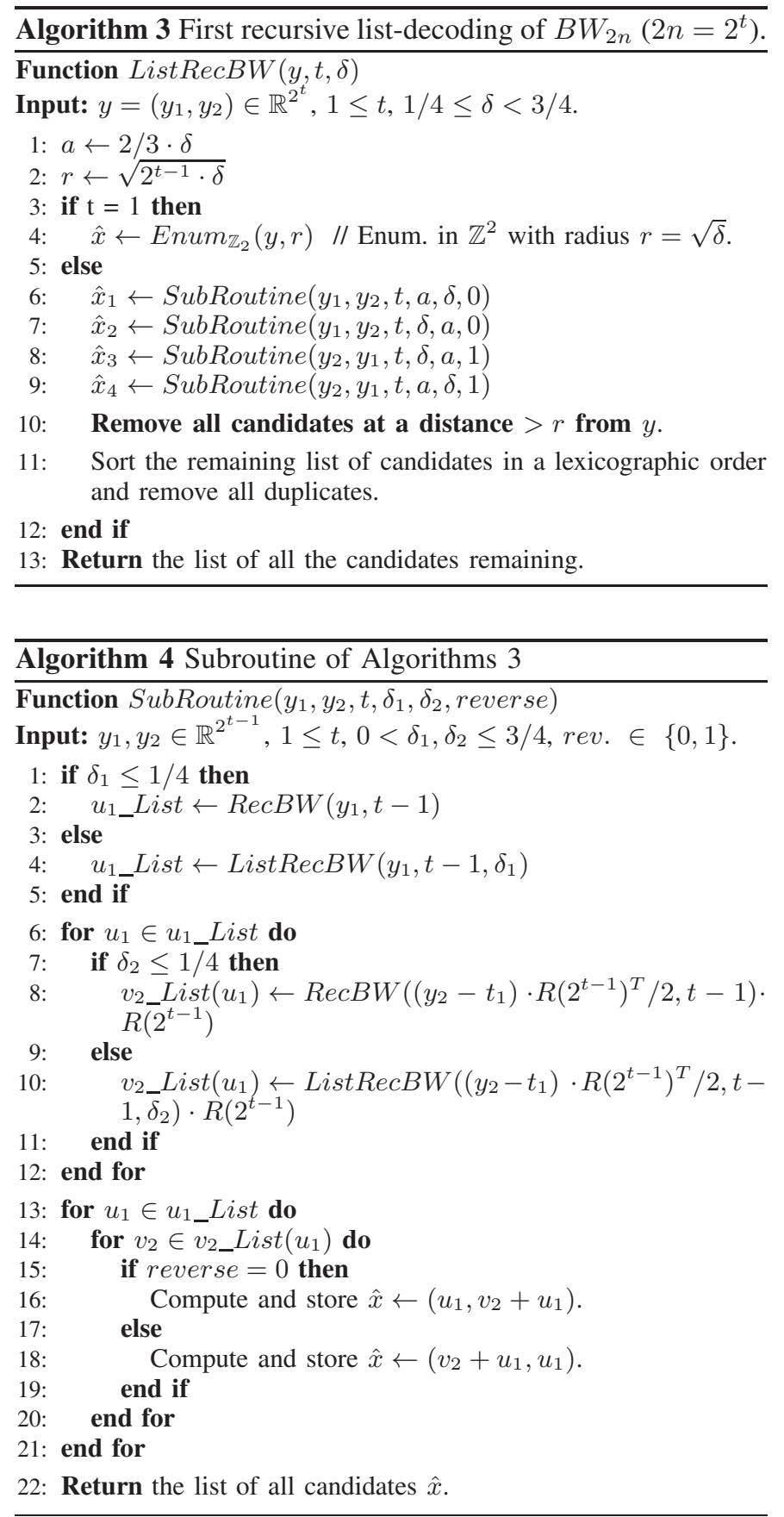

Unfortunately, the performance of Algorithm 3 on the Gaussian channel is disappointing. This is not surprising: notice that due to the "removing step" (10 in bold), some points that are correctly decoded by Algorithm 2 (the BDD) are not in the list outputted by Algorithm 3. Therefore, instead of removing all candidates at a distance greater than $r$, it is tempting to keep $\aleph$ candidates at each step.

\section{A. An efficient list decoder on the Gaussian channel}

We call Algorithm 5 a modified version of Algorithm 3 where the $\aleph(\delta)$ closest candidates are kept at each recursive step (instead of step 10, i.e. keeping only the points in the sphere of radius $r$ ) and steps 10 and 11 are flipped. The size of the list $\aleph(\delta)$, for a given $\delta$, is a parameter to be fine tuned: e.g. for $\delta=3 / 8$, one needs to choose only $\aleph(3 / 8)$ but for $\delta=1 / 2$, one needs to choose $\aleph(1 / 2)$ and $\aleph(2 / 3 \cdot 1 / 2=1 / 3)$. The following theorem follows from Theorem 3 .

Theorem 4. Given any point $y \in \mathbb{R}^{n}$ and $1 / 4 \leq \delta<9 / 16$, Algorithm 5 outputs the list of all lattice points in $B W_{n}$ lying within a sphere of relative squared radius $\delta$ around $y$ in time:

- $O\left(n^{2} \log n\right) \aleph(\delta)+O(n \log n) \aleph(\delta) \log \aleph(\delta)$ $1 / 4<\delta<3 / 8$

- $O\left(n^{\left.\frac{1+\log _{2}(1+\aleph(2 / 3 \delta))}{\log n} n\right) \aleph(\delta) \aleph(2 / 3 \delta)+}\right.$ $O\left(n^{\log _{2}(1+\aleph(2 / 3 \delta))}\right) \aleph(\delta) \log \aleph(\delta)$ if $3 / 8 \leq \delta<9 / 16$.

Note that on the Gaussian channel, the probability that $y$ is exactly between two lattice points is 0 . As a result, we can assume that $l(n, 1 / 4)=1$ and thus include $\delta=3 / 8$ in the first case of Theorem 4

As comparison, a similar modification of the algorithm of [14] would yield a complexity $O\left(n^{2}\right) \cdot \aleph(\delta)^{2}$. In the next section on simulations, we show that for $n=32$ and $n=64$, one should choose $\delta=3 / 8$ and $\aleph(\delta)$ should be at least 10 and 20, respectively. Moreover, for $n=128$ quasi-optimal performance is achieved for $\delta=1 / 2$ with $\aleph(\delta)=1000$ and $\aleph(2 / 3 \delta)=4$. With these parameters, our algorithm has a clear advantage thanks to the quasi-linear dependence in $\aleph(\delta)$.

\section{NUMERICAL RESULTS}

\section{A. Performance of Algorithm 5}

Figure 1 shows the influence of the list size when decoding $B W_{64}$ using Algorithm 5 with $\delta=3 / 8$. On this figure we also plotted an estimate of the MLD performance of $B W_{64}$, obtained as $\tau\left(B W_{64}\right) / 2 \cdot \operatorname{erfc}\left(\gamma /\left(8 \sigma_{\max }^{2} / \sigma^{2}\right)\right)$ [5, Chap. 3].

Figure 2 depicts the performance of Algorithm 5 for the $B W$ lattices up to $n=128$ and the universal bounds provided in [27] (see also [13] or [16], where it is called the sphere lower bound). These universal bounds are limits on the highest possible coding gain using any lattice in $n$ dimensions. For each $B W_{n}$ we tried to reduce as much as possible the list size while keeping quasi-MLD performance. The choice of $\delta=3 / 8$ yields quasi-MLD performance up to $n=64$ with small list size and thus reasonable complexity. This shows that $B W_{64}$, with Algorithm 5, is a good candidate to design finite constellations in dimension 64. However, for $n=128$ one needs to set $\delta=1 / 2$ and choose $\aleph(\delta)=1000$. Nevertheless, $\aleph(2 / 3 \cdot \delta)$ can be as small as 4 , which is still tractable. 


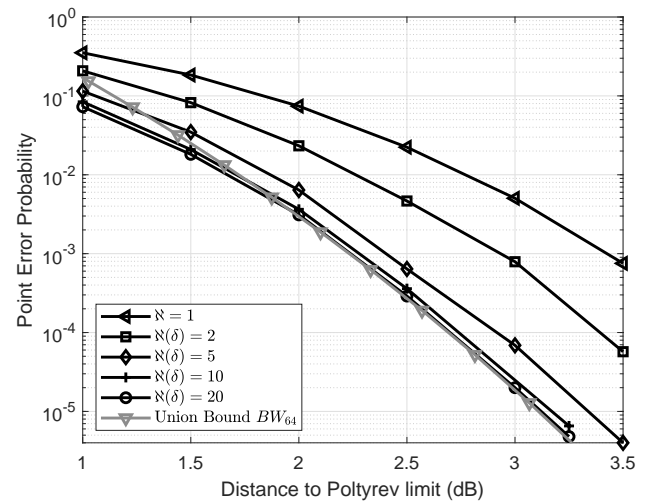

Fig. 1. Influence of the list size when decoding $B W_{64}$ using Alg. $5, \delta=3 / 8$.

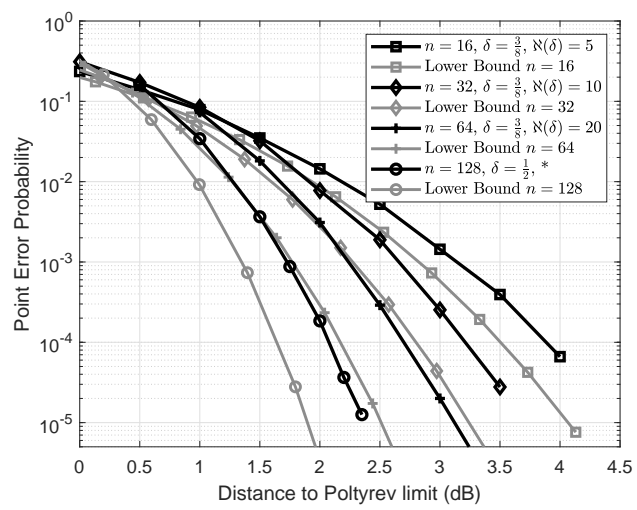

Fig. 2. Algorithm 5 for the $B W$ lattices up to $n=128$ and the universal bounds of [27]. * For $n=128, \aleph(\delta)=1000$ and $\aleph(2 / 3 \delta)=4$.

We compare these performances with existing schemes at $P_{e}=10^{-5}$. For fair comparison between the dimensions, we let $P_{e}$ be either the normalized error probability, which is equal to the point error-rate divided by the dimension (as done in e.g. [27]), or the symbol error-rate.

First, several constructions have been proposed for blocklengths around $n=100$ in the literature. In [18] a two-level construction based on $\mathrm{BCH}$ codes with $n=128$ achieves this error-rate at $2.4 \mathrm{~dB}$. The decoding involves an OSD of order 4 with 1505883 candidates. In [1] the multilevel (non-lattice packing) $\mathcal{S}_{127}(n=127)$ has similar performance but with much lower decoding complexity via generalized minimum distance decoding. In [23] a turbo lattice with $n=102$ and in [25] a LDLC with $n=100$ achieve the error-rate with iterative methods at respectively $2.75 \mathrm{~dB}$ and $3.7 \mathrm{~dB}$ (unsurprisingly, these two schemes are efficient for larger block-lengths). All these schemes are outperformed by $B W_{64}$, where $P_{e}=10^{-5}$ is reached at $2.3 \mathrm{~dB}$. Moreover, $B W_{128}$ has $P_{e}=10^{-5}$ at $1.7 \mathrm{~dB}$, which is similar to many schemes with block-length $n=1000$ such as the LDLC $(1.5 \mathrm{~dB})$ [25], the turbo lattice $(1.2 \mathrm{~dB})$ [23], the polar lattice with $n=1024(1.45 \mathrm{~dB})$ [28], and the LDA lattice $(1.27 \mathrm{~dB})$ [6]. This benchmark is summarized in Figure 3

\section{B. Performance of $B W$ finite constellations}

We uncover the performance of a Voronoi constellation [4] [10] based on the partition $B W_{64} / 2^{\eta} B W_{64}$ via Monte Carlo simulation, where $\eta$ is the desired rate in bits per channel

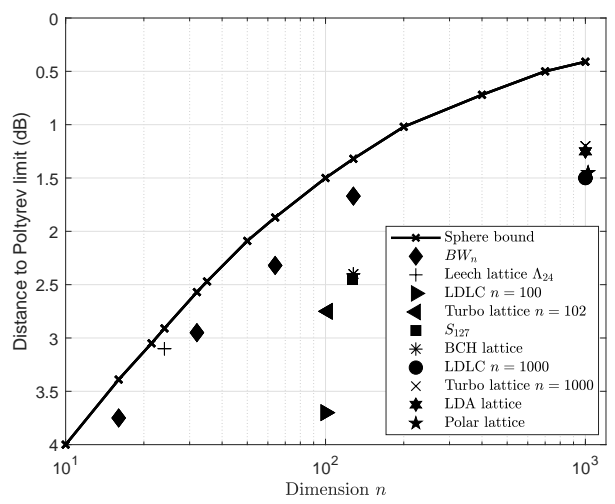

Fig. 3. Perf. of different lattices for normalized error probability $P_{e}=10^{-5}$.

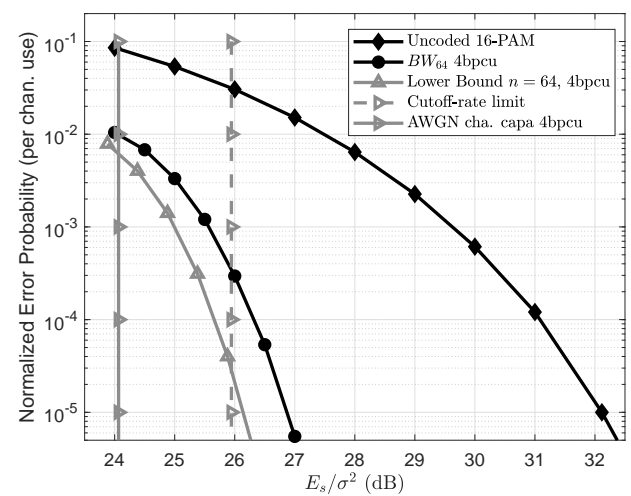

Fig. 4. Performance of a Voronoi constellation based on the partition $B W_{64} / 2^{4} B W_{64}$ where Algorithm 5, with $\delta=3 / 8$ and $\aleph(\delta)=20$, is used for encoding and decoding. The cutoff-rate limit is $1.7+0.179 \mathrm{~dB}$ right to Shannon limit (coding + shaping loss for $n=64$ ) [12].

use (bpcu): i.e. both the coding lattice and the shaping lattice are based on $B W_{64}$. It follows that the encoding complexity is the same as the decoding complexity: the complexity of Algorithm 5 with $\delta=3 / 8$ and $\aleph(\delta)=20$. Figure 4 exhibits the performance of our scheme for $\eta=4$ bpcu. In our simulation, the errors are counted on the uncoded symbols. The error-rate also includes potential errors due to incomplete encoding, which seem to be negligible compared to decoding errors. Again, we plotted the best possible performance of any lattice-based constellation in dimension 64 (obtained from [27]). The scheme performs within $0.7 \mathrm{~dB}$ of the bound.

\section{Conclusions}

Our recursive paradigm can be seen as a tree search algorithm and our decoders fall therefore in the class of sequential decoders. While the complexity of Algorithm 5 remains stable and low for $n \leq 64$, there is a significant increase for $n=128$ and it becomes intractable for $n=256$ due to larger lists. This is not surprising from the cut-off rate perspective [12]; For $n=64$ the MLD is still at a distance of $1 \mathrm{~dB}$ from this limit (Figure 4), but it is very close to the limit for $n=128$ and potentially better at larger $n$. One should not expect to perform quasi-MLD of these lattices with any sequential decoder. This raises the following open problem: can we decode lattices beyond the cut-off rate in nonasymptotic dimensions, i.e. $n<300$, where classical capacityapproaching decoding techniques (e.g. BP) cannot be used? 


\section{APPENDIX}

\section{A. Analysis of the effective error coefficient}

Let us define the decision region of a BDD algorithm $\mathbb{R}_{B D D}(\mathbf{0})$ as the set of all points of the space that are decoded to $\mathbf{0}$ by the algorithm. The number of points at distance $\rho(\Lambda)$ from the origin that are not necessarily decoded to $\mathbf{0}$ are called boundary point of $\mathbb{R}_{B D D}(\mathbf{0})$ The number of such points is called effective error coefficient of the algorithm. The performance of BDD algorithms are usually estimated via this effective error coefficient [11] [24]. Indeed, BDD up to the packing radius achieves the best possible error exponent on the Gaussian channel, but the performance might be significantly degraded, compared to MLD, due to a high effective error coefficient.

In [19], the error coefficient of the parallel decoder is not computed and the performance of the algorithm is not assessed on the Gaussian channel. The following analysis of Algorithm 2 is also valid for the parallel decoder [19]. Let us express the point to be decoded as $y=x+\eta$, where $x \in B W_{n}$ and $\eta$ is a noise pattern. Scale $B W_{n}$ such that its packing radius is 1 . It is easily seen that any $\eta$ of the form $\left( \pm \frac{1}{\sqrt{2^{t}}} 2^{t}\right)=\left( \pm \frac{1}{\sqrt{2^{t}}}, \ldots, \pm \frac{1}{\sqrt{2^{t}}}\right), t=\log _{2}(n)$, is on the boundary of $\mathbb{R}_{B D D}(\mathbf{0})$. The number of such noise patterns is $2^{2^{t}}=2^{n}$. According to Forney's rule of thumb, every factorof-two increase in the number of nearest neighbor results in a $0.2 \mathrm{~dB}$ loss in effective coding gain [12]. Since the kissing number of $B W_{n}$ is $\prod_{i=1}^{t}\left(2^{i}+2\right) \approx 4.768 \ldots \cdot 2^{0.5 \log _{2} n\left(\log _{2} n+1\right)}$ [5], to be compared to the above number of noise patterns $2^{n}$, we see that the loss in performance compared to MLD (in $\mathrm{dB}$ ) is expected to grow as $\approx 0.2 n$. However, this rule holds only if the effective error coefficient is not too large and the performance of Algorithm 2 is not as bad in practice. Nevertheless, this analysis hints that one should expect the performance of this BDD to degrade as $n$ increases.

\section{B. The Merge Sort Algorithm}

Let $l^{k, n}=\left(x_{1}^{n}, x_{2}^{n} \ldots, x_{k}^{n}\right)$ be a list of $k$ elements $x$ of dimension $n$ (assume for the sake of simplicity that $k$ is a power of 2). This list can be split into two lists of equal size $l_{1}^{k / 2, n}$ and $l_{2}^{k / 2, n}$ and we write $l^{k, n}=\left(l_{1}^{k / 2, n}, l_{2}^{k / 2, n}\right)$.

Then, we define the function Merge as a function that takes two sorted lists of $k$ elements $x$ of dimension $n$ as input (as well as $k$ and $n$ ) and returns a unique sorted list of the $2 k$ elements. There exists several variants of this function, but the complexity is always $O(n \cdot k)$.

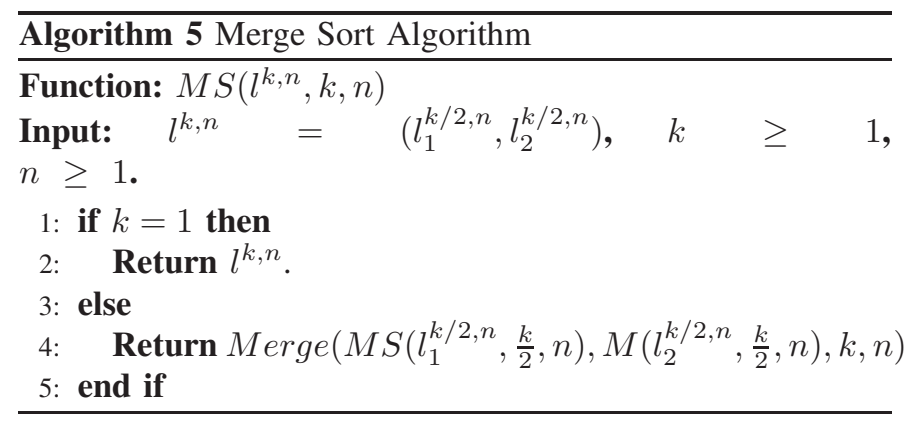

Let $\mathfrak{C}(k, n)$ be the complexity of the $M S$ function (Algorithm 6). The complexity of this algorithm is $\mathfrak{C}(k, n)=$ $2 \mathfrak{C}(k / 2, n)+O(k \cdot n)=O(k \log (k) \cdot n)$

\section{REFERENCES}

[1] D. Agrawal and A. Vardy, "Generalized minimum distance decoding in euclidean space: Performance analysis," IEEE Trans. Inform. Theory, vol. 46, pp. 6083, 2000.

[2] E. S. Barnes and G. E. Wall, "Some extreme forms defined in terms of Abelian groups," J. Australian Math. SOC., vol. 1, pp. 47-63, 1959.

[3] M. F. Bollauf, R. Zamir, S. I. R. Costa, "Multilevel Constructions: Coding, Packing and Geometric Uniformity," IEEE Trans. on Inform. Theory, Vol. 65, 2019.

[4] J. Conway and N. Sloane, "A fast encoding method for lattice codes and quantizers," IEEE Trans. Inform. Theory, vol. 19, pp. 820-824, 1983.

[5] J. Conway and N. J. A. Sloane. Sphere packings, lattices and groups. Springer-Verlag, New York, 3rd edition, 1999.

[6] N. di Pietro, J. J. Boutros, G. Zmor, L. Brunel, "Integer Low-Density Lattices based on Construction A", 2012 IEEE Information Theory Workshop, 2012.

[7] I. Dumer and K. Shabunov, "Soft-decision decoding of Reed-Muller codes: recursive lists," IEEE Trans. Inform. Theory, vol. 52, pp. 12601266,2006

[8] N. D. Elkies, "Mordell-Weil lattices in characteristic 2: III. A MordellWeil lattice of rank 128," Experimental Math., vol. 3, pp. 467-473, 2001.

[9] G. D. Forney, Jr., "Coset codes II: Binary lattices and related codes," IEEE Trans. Inform. Theory, vol. 34, pp. 1152-1187, 1988.

[10] G. D. Forney, Jr., "Multidimensional Constellations - part II: Voronoi Constellations," IEEE J. Select. Areas Com., vol. 7, pp. 941-958, 1989.

[11] G. D. Forney, Jr. and A. Vardy, "Generalized Minimum-Distance decoding of Euclidean-Space Codes and Lattices," IEEE J. Select. Areas Com., vol. 42, pp. 1992-2026, 1996.

[12] G. D. Forney and G. Ungerboeck, "Modulation and Coding for Linear Gaussian Channels," IEEE Trans. Inform. Theory, vol. 44, pp. 2384$2415,1998$.

[13] G. D. Forney, M. D. Trott, and S. Chung, "Sphere-Bound-Achieving Coset Codes and Multilevel Coset Codes," IEEE Trans. Inform. Theory, vol. 46, pp. 820-850, 2000 .

[14] E. Grigorescu and C. Peikert, "List-Decoding Barnes-Wall Lattices," Computational Complexity, vol. 26, pp 365-39, 2017.

[15] J. Harshan, E. Viterbo, and J.-C. . Belfiore, "Practical Encoders and Decoders for Euclidean Codes from Barnes-Wall Lattices," IEEE Trans. Communications, vol. 61, pp. 4417-4427, 2013.

[16] A. Ingber, R. Zamir, and M. Feder, "Finite-Dimensional Infinite Constellations," IEEE Trans. Inform. Theory, vol. 59, pp. 1630-1656, 2013.

[17] F. J. MacWilliams and N. J. A. Sloane, The Theory of Error-Correcting Codes. Amsterdam, The Netherlands: North-Holland, 1977.

[18] T. Matsumine, B. M. Kurkoski, and H. Ochiai, "Construction D Lattice Decoding and Its Application to BCH Code Lattices," 2018 IEEE Global Communications Conference (GLOBECOM), 2018.

[19] D. Micciancio and A. Nicolosi, "Efficient Bounded Distance Decoders for Barnes-Wall Lattices," 2008 IEEE Int. Symp. Inform. Theory, 2008.

[20] G. Nebe, "An even unimodular 72-dimensional lattice of minimum 8," J. Reine Angew. Math., vol. 673, pp. 237-247, 2012.

[21] M. Plotkin, "Binary codes with specified minimum distances," IEEE Trans. Inform. Theory, vol. 6, pp. 445-450, 1960.

[22] G. Poltyrev, "On coding without restrictions for the AWGN channel," IEEE Trans. Inform. Theory, vol. 40, pp. 409-417, 1994.

[23] A. Sakzad, M. Sadeghi, and D. Panario, Turbo Lattices: Construction and Performance Analysis, arXiv preprint arXiv:1108.1873 2011. 48th Annual Allerton Conf. on Com., Control, and Computing , 2011.

[24] A. J. Salomon and O. Amrani, "Encoding and Decoding Binary Product Lattices," IEEE Trans. Inform. Theory, vol 52, pp 5485-5495, 2006.

[25] N. Sommer, M. Feder, and O. Shalvi, "Low-density Lattice Codes," IEEE Trans. Inform. Theory, vol. 54, pp. 1561-1585, 2008.

[26] G. Schnabl and M. Bossert, "Soft-decision decoding of Reed-Muller codes as generalized multiple concatenated codes," IEEE Trans. on Inform. Theory, vol. 41, pp. 304-308, 1995.

[27] V. Tarokh, A. Vardy, and K. Zeger, "Universal bound on the performance of lattice codes," IEEE Trans. Inform. Theory, vol. 45, pp. 670-681, 1999.

[28] Y. Yan, C. Ling, X. Wu, "Polar lattices: Where Arkan meets Forney," 2013 IEEE Int. Symp. Inform. Theory, 20013. 